\title{
Basic Understanding of Temporomandibular Joint and Its Dysfunction among Undergraduate Students-A Survey Report
}

\author{
Shilpa Dandekeri ${ }^{1}$ Pooja B. Kavassery ${ }^{1} \quad$ Chethan Hegde $^{1} \quad$ Sowmya M. Kumar ${ }^{1} \quad$ Bharathraj Shetty ${ }^{1}$
}

${ }^{1}$ Department of Prosthodontics and Crown and Bridge, A.B. Shetty Memorial Institute of Dental Sciences, Mangaluru, Karnataka, India
Address for correspondence Pooja B. K., MDS (Student), Department of Prosthodontics and Crown \& Bridge, A.B. Shetty Memorial Institute of Dental Sciences, Medical Sciences Complex, Deralakatte, Mangaluru 575018, Karnataka, India (e-mail: drpbk92@gmail.com).

Abstract

Keywords
- temporomandibular
joint
- temporomandibular
disorder
- orofacial pain

Background Temporomandibular disorder is the most commonly occurring type of orofacial pain. A general dental practitioner must be able to recognize and distinguish pain related to the joint to be capable of providing treatment or send for a referral. This survey was undertaken to test the undergraduate students in their residency program to analyze their skills in basic understanding and diagnostic capability relevant to temporomandibular joint (TMJ) disorder.

Materials and Methods A total of 110 dental students undergoing their residency program in the institute of A.B. Shetty Memorial Institute of Dental Sciences were selected. They were given a questionnaire pro forma to fill out which included questions regarding the anatomy, physiology, pathology, diagnosis, and treatment plan of TMJ disorders.

Results Note that $71.75 \% \pm 6.13 \%$ of students answered anatomy questions correctly, $46.6 \% \pm 28 \%$ answered physiology questions correctly, $34.67 \% \pm 5.5 \%$ answered pathology questions correctly, $23.67 \% \pm 2.5 \%$ answered diagnosis questions correctly, and $27.25 \% \pm 15.8 \%$ answered questions of treatment plan correctly. Note that $100 \%$ of them understood the importance of TMJ in dentistry.

Conclusion This shows that there is an awareness about the basic understanding of the TMJ; however, to be able to clinically apply the knowledge and diagnose a disorder of TMJ, more than half of the participants were unacquainted of it and the curriculum should stress on improvement of this understanding at the undergraduate level itself.

\section{Introduction}

Temporomandibular joint (TMJ) functioning involves a complex mechanism of the muscles of mastication, ligaments, and the bones of the joint which when work in a synchrony bring about fluidity in the movement of the jaw for its various activities. Owing to this, a small flaw in these mechanisms can overstress a particular area and bring about a compensatory change in the function to meet the demands. Depending on the threshold of the patients and the severity, these compensatory changes may be perceived as a discomfort, disability, and restriction in function. ${ }^{1}$
Temporomandibular disorders (TMDs) are considered to be the most commonly occurring type of orofacial pain. ${ }^{2}$ Patients who report presenting with TMD may not complain of tenderness or discomfort specific to the area of the joint. Hence, appropriate understanding of the basic physiology and pathology of the TMJ is needed to diagnose the pain or disorder to be related to the joint and plan the treatment or send for an appropriate referral.

Undergraduate students who move on to be general dental practitioners require this basic understanding to recognize and diagnose a case pertaining to the joint and also understand the treatment plan or refer to a specialist in the field.
License terms

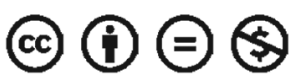


Hence, this present survey was undertaken to understand the awareness among undergraduate students undergoing their residency program regarding the anatomy, physiology, pathology, diagnosis, and treatment pertaining to the TMJ.

\section{Materials and Methods}

For this institutional survey, 110 dental students undergoing their residency program in the institute of A.B. Shetty Memorial Institute of Dental Sciences were selected. They were given a questionnaire pro forma to fill. The anatomy, physiology, pathology, diagnosis, and treatment plan of TMJ disorders were questioned. The results were analyzed and based on the collective response of each question. Percentage of correct responses of each question was calculated. The results were further grouped under different sections anatomy (Q1-Q4), physiology (Q5-Q9), pathology (Q10-Q12), diagnosis (Q13Q15), treatment planning (Q16-Q19), and attitude (Q20).

\section{Results}

The results were analyzed using IBM SPSS Statistics Version 22 (IBM Corp., Armonk, New York, United States). Number of students giving correct answer was identified and the percentage was calculated. These results are presented in bar diagrams (-Figs. 1-20). The results were further grouped under sections of anatomy (Q1-Q4), physiology (Q5-Q9), pathology (Q10-Q12), diagnosis (Q13-15), treatment

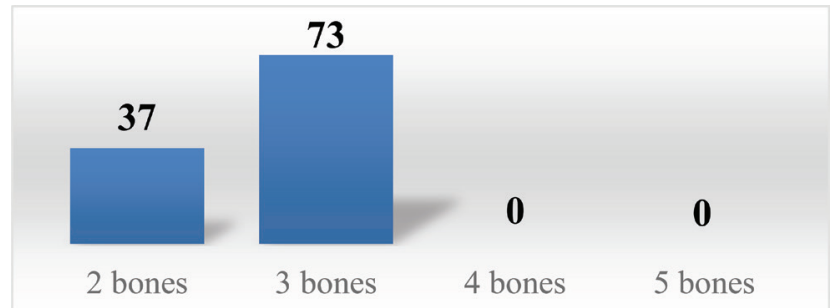

Fig. 1 How many bones is the Temporomandibular Joint made up of?

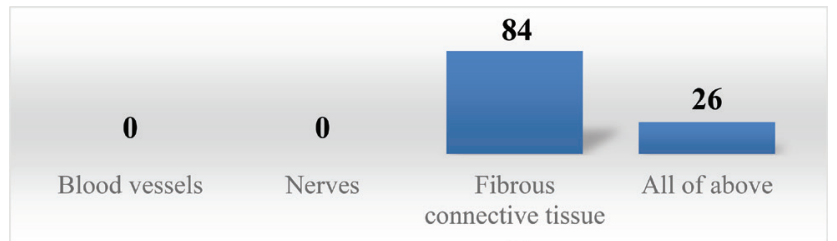

Fig. 2 What is the Articular disc made up of?

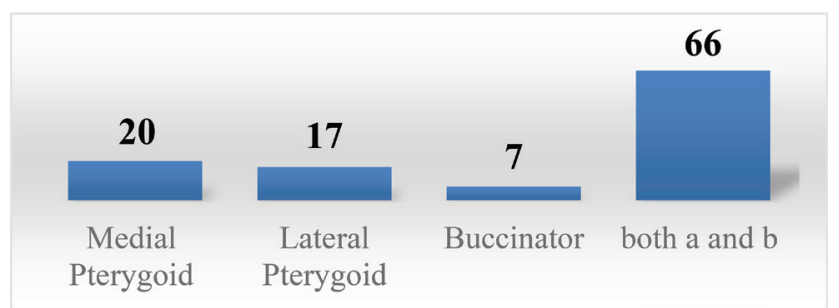

Fig. 3 Which is/are the Muscles of Mastication?

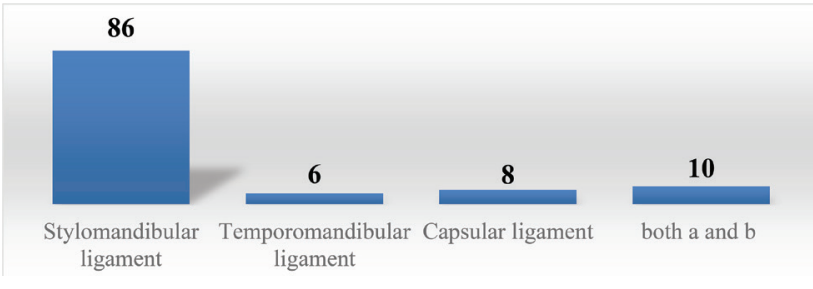

Fig. 4 Which is/are the accessory ligaments of temporomandibular joint?

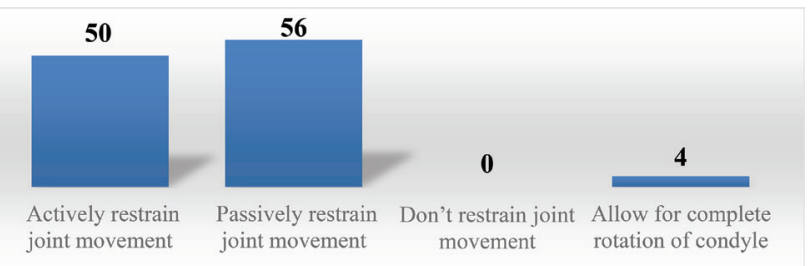

Fig. 5 What is the function of Ligaments of temporomandibular joint?

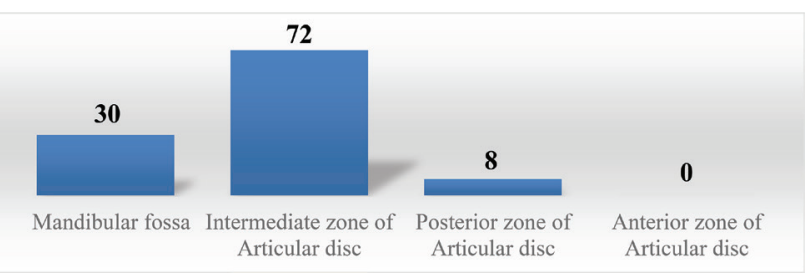

Fig. 6 What does the condyle articulate with during normal function?

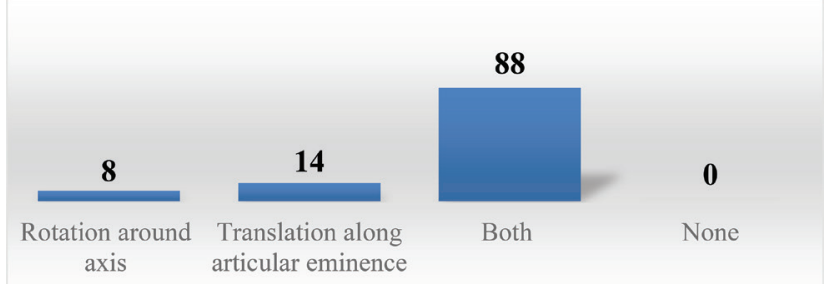

Fig. 7 Which movement is seen in the temporomandibular joint?

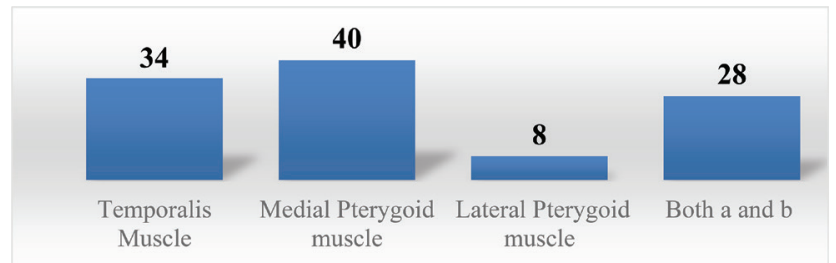

Fig. 8 Which muscle aids closing of mouth?

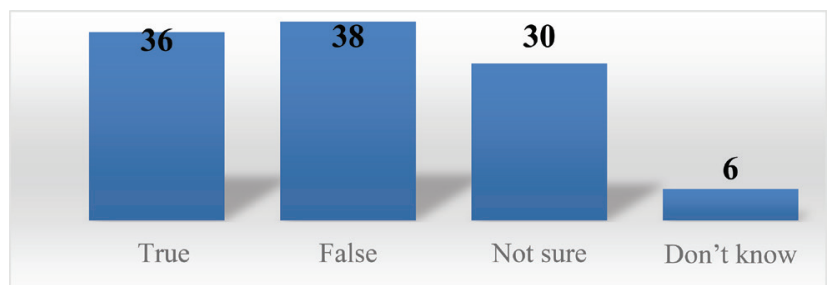

Fig. 9 Which muscle aids the opening of mouth? 


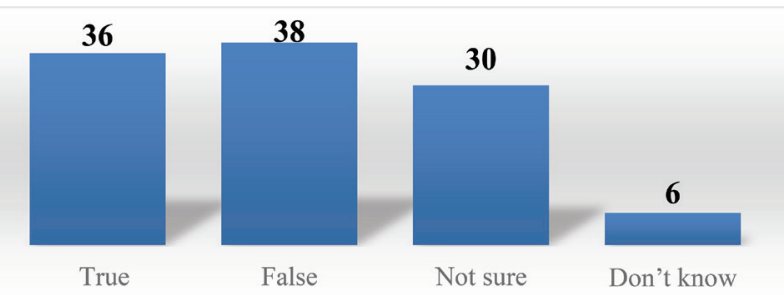

Fig. 10 Disorders of the temporomandibular joint is seen only with temporal bone, condyle and disc.

\section{8}

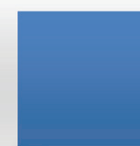

Restricted mouth opening

Fig. 11 Which symptoms can be presented as a temporomandibular joint disorder?

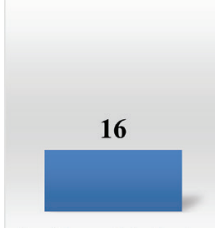

Locking of Articular disc

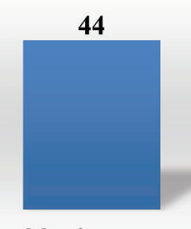

restricti
Muscle spasm

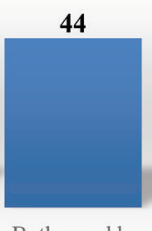

Both $a$ and $b$

\section{2}

0

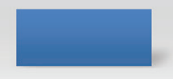

All of above
Fig. 12 What can cause restriction in mouth opening?

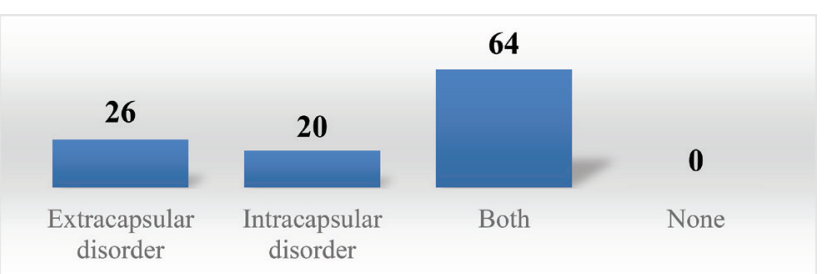

Fig. 13 What is a patient presenting with acute pain on right side of the angle of the mandible after class II cavity restoration in 46 likely to have?

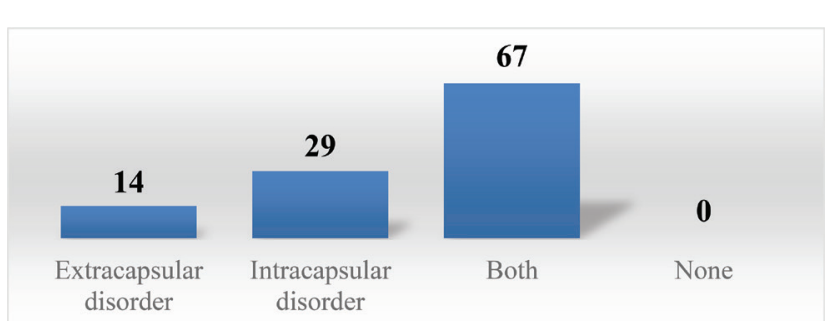

Fig. 14 What is a patient presenting with a click sound while opening mouth likely to have?

planning (Q16-Q19), and attitude (Q20), and the calculated percentage of correct answers of each question were presented in a cluster bar diagram ( - Fig. 21). Mean and standard deviation of the percentage value was computed according to the sections of the questionnaire. Note that $71.75 \% \pm 6.13 \%$ of students answered anatomy questions

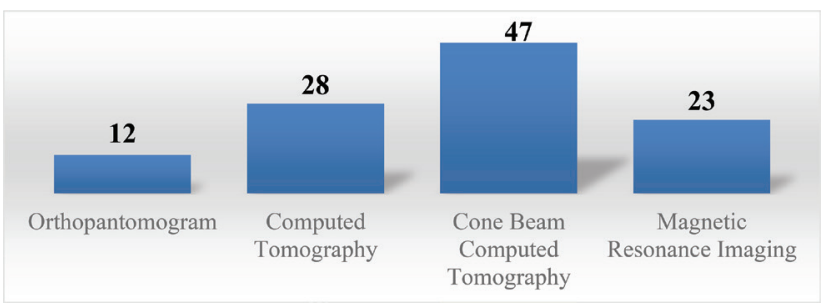

Fig. 15 Which scan provides the best radiologic view for examination of temporomandibular joint?

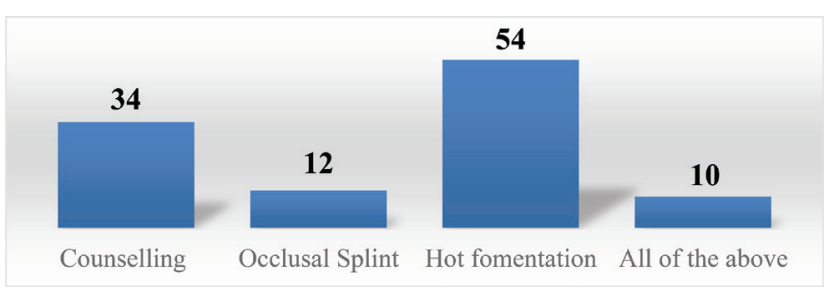

Fig. 16 What is the treatment for disorders of temporomandibular joint?

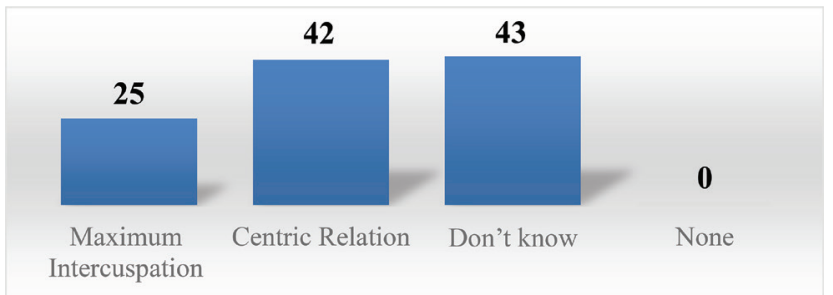

Fig. 17 Which occlusal relation is ideal for restoration of single tooth in complete dentate patient?

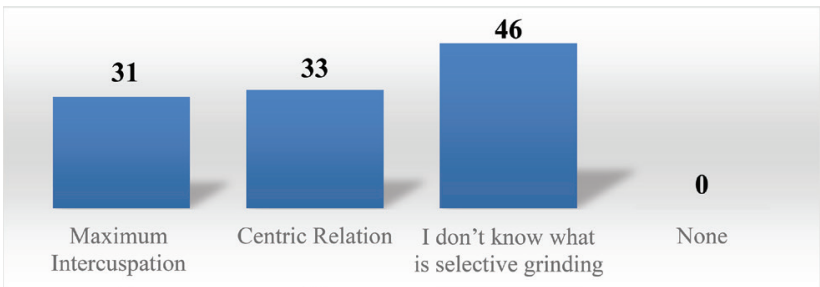

Fig. 18 Which occlusal relation is advocated for selective grinding of teeth as a treatment to treat pain in temporomandibular joint?

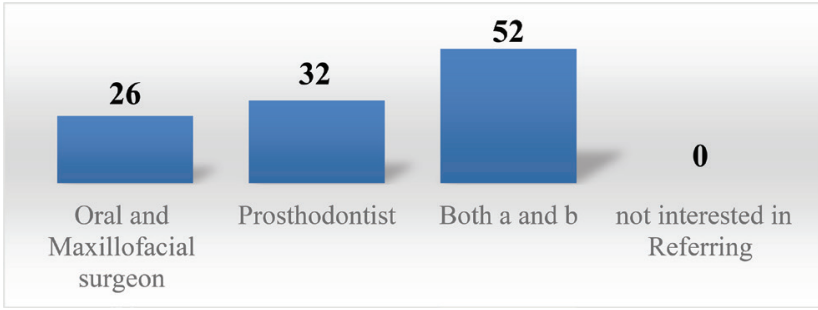

Fig. 19 Who would you consult when a patient presents to you with a temporomandibular joint abnormality?

correctly, $46.6 \% \pm 28 \%$ answered physiology questions correctly, $34.67 \% \pm 5.5 \%$ answered pathology questions correctly, $23.67 \% \pm 2.5 \%$ answered diagnosis questions correctly, and $27.25 \% \pm 15.8 \%$ answered questions of treatment plan correctly. Note that $100 \%$ of them understood the importance of TMJ in dentistry. 


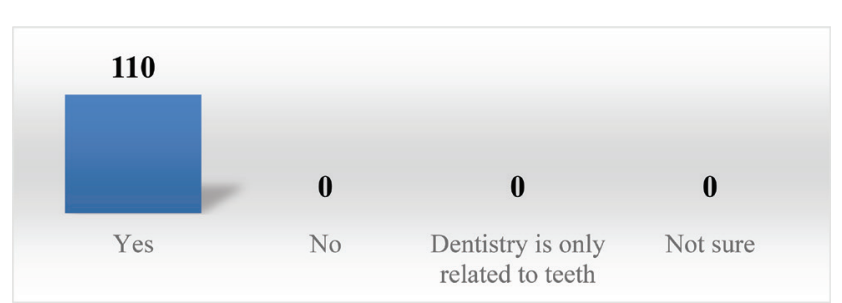

Fig. 20 Do you feel that understanding the function and dysfunction of temporomandibular joint is necessary to a dentist? that it is made up of blood vessels, nerves, and fibrous connective tissue.

Medial and lateral pterygoid are two of the muscles of mastication, to this 66 (60\%) of the total students answered correctly, while 20 of them answered to be medial pterygoid, 17 of them answered to be lateral pterygoid, and 7 of them answered it to be buccinator.

Accessory ligaments of the TMJ are stylomandibular and sphenomandibular ligament, to this $86(78.1 \%)$ of the total

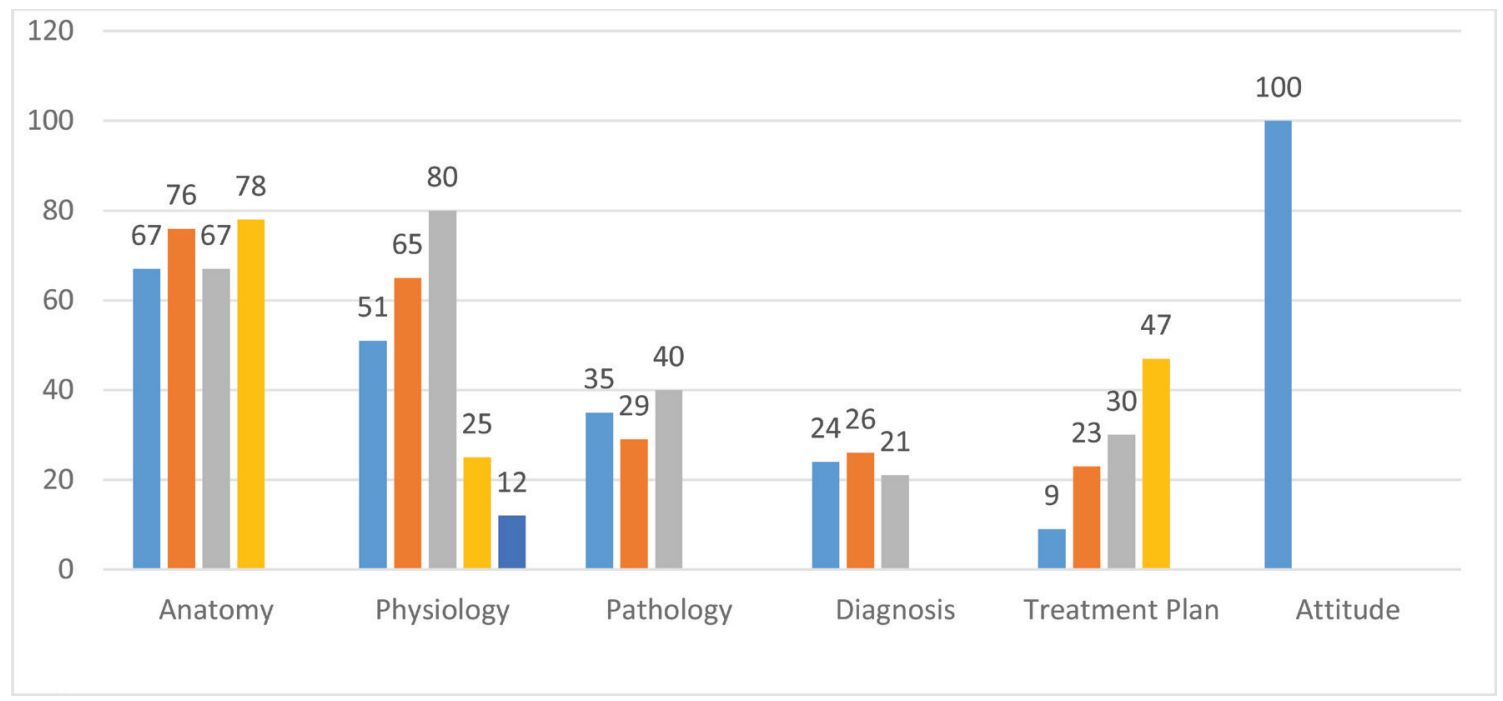

Fig. 21 Percentage of correct answers for each question.

\section{Discussion}

Being the most common cause of orofacial pain, ${ }^{2}$ importance of TMJ and its disorders must be stressed at the undergraduate level. The present study was conducted to analyze the knowledge of students undergoing their undergraduate residency program regarding their fundamental understanding of TMJ and its disorders. Results of studies ${ }^{3-5}$ conclude that $^{-1}$ the knowledge and attitude of dentists toward treating a TMD is undesirable.

The present study included a total of 110 students who completed the questionnaire regarding different aspects of TMJ disorders.

In this study, knowledge-based awareness about different aspects of TMJ was analyzed by distributing questionnaire with questions pertaining to different sections of anatomy, physiology, pathology, diagnosis, treatment plan, and attitude. ${ }^{1,6,7}$ Correct response of each question was calculated in percentage. The percentages were further subgrouped under different sections of questions.

Out of 110 study participants, only 73 students (66.3\%) answered correctly that TMJ is made up of 3 bones comprising of the condylar head, glenoid fossa of the temporal bone, and articular disc that is regarded as nonossified bone, while 37 answered that it is made up of 2 bones.

Eighty-four (76.3\%) of total students answered correctly that the articular disc is an avascular tissue made up of fibrous connective tissue, while the remaining 26 answered students answered correctly, while 6 answered that it is the temporomandibular ligament, 8 answered it to be the capsular ligament, and 10 answered that it is both stylomandibular and temporomandibular ligament.

Ligaments of the TMJ passively restrain the movement and only 56 (50.9\%) of them answered correctly. Fifty students answered that the ligaments function by actively restraining the movement of the joint while 4 of them said that it allows for complete rotation of the condyle.

Condyle articulates with the intermediate zone of the articular disc, to this 72 (65.4\%) of the students answered correctly, while 30 students answered that the condyle articulates with the mandibular fossa and 8 with the posterior zone of the articular disc.

TMJ movement is seen as both rotatory and translatory motion, to this 88 (80\%) students answered correctly while 8 believed that the movement is only rotation around the axis and 14 believed that the movement is only as translation along the articular eminence.

Closing of the mouth is aided by the elevator muscles. Both the temporalis muscle and medial pterygoid muscle are elevator muscles and 28 (25.4\%) answered correctly. Thirty-four believed that only the temporalis muscle functions to close the mouth, while 40 answered it to be only medial pterygoid. Eight of the total students answered it to be lateral pterygoid muscle.

Opening of the mouth is aided by the depressor muscles. Both the lateral pterygoid muscle and suprahyoid muscles form the depressors and only 14 (12.7\%) of the total students 
answered correctly. Eighty of the total students believed that only lateral pterygoid functions to open the mouth, while 16 of them answered it to be the medial pterygoid muscle.

Disorders of the TMJ encompass the muscles, ligaments, temporal bone, condyle, and articular disc. To this 38 (34.5\%) answered correctly that the joint disorder does not pertain only to the temporal bone, condyle, and the articular disc, while 36 believed that it is true. Thirty students were not sure while 6 students did not know.

Thirty-two (29\%) students correctly answered that TMJ disorder can be presented with restricted mouth opening, pain at the region of teeth, and temple, while the remaining 78 believed that it only presented with restricted opening of the mouth.

Restricted mouth opening can be due to both reasons of spasm of the muscles and locking of the articular disc. Forty-four (40\%) answered correctly, 44 believed it to be muscle spasm, and 16 believed locking of the articular disc restricts the opening of mouth. Six answered that lengthening of the muscle can restrict opening of mouth.

Acute pain following a cavity restoration near the angle of the mandible is a muscular disorder. To this 26 (23.6\%) answered correctly with extracapsular disorder, while 64 believed it is due to both extracapsular disorder and intracapsular disorder and 20 believed it is due to intracapsular disorder.

A click sound is an indication of displacement of the articular disc which comes under intracapsular disorders. Twenty-nine (26.3\%) students answered correctly, while 67 students believed that it is both intracapsular and extracapsular disorder and 14 students believed that it is an extracapsular disorder.

Magnetic resonance imaging is the gold standard for the diagnosis of TMJ disorder, to this 23 (20.9\%) of the total students answered correctly. Note that 47, 28, and 12 students believed that radiologic examination of TMJ is best viewed in cone beam computed tomography, computed tomography, and orthopantomogram, respectively.

Disorders of the TMJ can be treated with counseling, occlusal splint, and hot fomentation. Only 10 (9\%) of the total students answered correctly, while 54, 34, and 12 of the total students believed that only hot fomentation, counseling, and occlusal splint, respectively, treat TMJ disorder.

Restoration of a single tooth in a complete dentate patient must always be done in maximum intercuspation. Only $25(22.7 \%)$ of the total students answered correctly, while 42 believed it should be done in centric relation and 43 did not know.

Selective grinding should be done in centric relation. Thirty-three (30\%) of the total students answered correctly, while 31 believed that it should be done in maximum intercuspation and 46 students did not know what is selective grinding.

Both prosthodontists and oral and maxillofacial surgeons play a vital role in the treatment of TMJ. Fifty-two (47.2\%) answered correctly, while 32 and 26 believed that prosthodontists alone or oral and maxillofacial surgeons alone, respectively, treat an abnormality of TMJ.

All students answered that the understanding of function and dysfunction of the TMJ is necessary for a dentist.
The level of awareness decreased as we progressed from the first to last section. The highest percentage of awareness was calculated in the section of anatomy followed by physiology. The mean calculated percentage of sections of pathology, diagnosis, and treatment planning were below $50 \%$ indicating that less than half of the study participants were not aware of these aspects, thus proving the lack of knowledge in these aspects.

This shows that there is an awareness about the basic understanding of the TMJ; however, to be able to clinically apply the knowledge and diagnose a disorder of TMJ, more than half of the participants were unacquainted of it. This indicates the lack of practical knowledge and clinical translation of knowledge among the undergraduate students. This is in accordance to a study conducted by Alhussini et al. ${ }^{3}$

In the present study, $100 \%$ of the students realized the importance of TMJ for a dentist. Treatment planning of TMJ is an interdisciplinary approach between a prosthodontist and an oral and maxillofacial surgeon. However, when confronted with a patient with TMJ disorder, the general dental practitioner must be able to differentiate it from the other causes of orofacial pain and be able to diagnose the type of disorder and also be able to educate the patient and counsel them regarding the physical therapeutic modalities and further refer them to a specialist.

However, from the present study, it can be inferred that the curriculum of the undergraduate level does not stress on covering all the aspects related to TMJ and hence its importance must be stressed.

\section{Conclusion}

Awareness of the diagnosis and treatment planning of TMDs is of paramount importance in the dental field to be able to provide effective treatment and good quality of life to the patient. This knowledge must be imparted to the students in their undergraduate course which will result in a welltrained dentist.

From the present survey that was conducted, the following conclusions can be drawn: $71.75 \%$ of students answered anatomy questions correctly, $46.6 \%$ answered physiology questions correctly, $34.67 \%$ answered pathology questions correctly, 23.67\% answered diagnosis questions correctly, and $27.25 \%$ answered questions of treatment plan correctly. The knowledge required to apply in a clinical scenario was deficient.

\section{Conflict of Interest}

None.

\section{References}

1 Okeson J. Management of Temporomandibular Disorders and Occlusion. 7th ed. Elsevier; 2013

2 Bell W, Temporomandibular Disorders, Classification, Diagnosis and Treatment. 3rd ed. London: Yearbook Medical Publisher; 2019

3 AlhussiniDA,MominkhanDM,AlhamedFJ,SaklouRA,Abdel-Alim HA. Prevalence and awareness of temporomandibular joint 
disorders among patients in King Abdulaziz University, Dental Hospital. J Dent Health Oral Disord Ther 2017;8(5):00300

4 Choudhary SH, Kale LM, Mishra SS. Sodhi S, Muley PB, Pandey ND. An institutional survey for knowledge-based and self-awareness assessment in temporomandibular joint disorders among dental students. Indian. J Dent Res 2016;27(3):262-267
5 Tegelberg A, Wenneberg B, List T. General practice dentists' knowledge of temporomandibular disorders in children and adolescents. Eur J Dent Educ 2007;11(4):216-221

6 Dawson P, Functional Occlusion. St. Louis, MO: Mosby; 2007

7 Chaurasia B, B.D. Chaurasia's Human Anatomy. New Delhi, India: CBS Publishers \& Distributors; 2004 